\title{
PUBLIC HEALTH EDUCATION IN MEDICAL SCHOOLS - THE IMPACT OF THE COVID-19 PANDEMIC
}

\author{
Veena C. Rodrigues ${ }^{\mathrm{a} *}$, Eleanor J. Hothersall ${ }^{\mathrm{b} *}$, \\ and Marc Davies ${ }^{\mathrm{c} *}$
}

Corresponding Author: Professor Veena C. Rodrigues, Norwich Medical School, University of East Anglia, Norwich NR4 7TJ, UK; email: v.rodrigues@uea.ac.uk

The COVID-19 pandemic has brought the speciality of public health (PH) into the spotlight, creating interest from students and curriculum designers that was previously unimagined. The relevance of teaching about infectious diseases, pandemics and health inequalities has a new resonance for many. It is perhaps ironic that this demand for specialist input has come at the same time that the $\mathrm{PH}$ workforce find themselves under increased pressure leading and managing the response to the pandemic within local communities.

$\mathrm{PH}$ and related social sciences have historically been undervalued and underfunded in medical education within the UK (Gillam and Bagade, 2006). Although guidance from the General Medical Council (GMC) to UK medical schools (GMC, 2018) specifies that 'newly qualified doctors must be able to apply the principles, methods and knowledge of population health and the improvement of health and sustainable healthcare to medical practice,' in practice, the depth and breadth of $\mathrm{PH}$ in medical curricula varies across medical schools

a Professor of Public Health and Medical Education, Norwich Medical School, University of East Anglia

${ }^{\mathrm{b}}$ Head of MBChB and Associate Director of Public Health, University of Dundee/NHS Tayside

c Specialty Registrar in Public Health, Public Health Wales

* Co-Chairs, Public Health Educators in Medical Schools (PHEMS) Special Interest Group, Faculty of Public Health, UK 
(Gillam, Rodrigues, and Myles, 2015). Medical schools are expected to demonstrate that their curricula enable the achievement of 11 professional knowledge learning outcomes on health promotion and illness prevention (Box 1), as well as several other related outcomes such as research methods and social sciences (GMC, 2018).

Box 1. GMC outcomes for graduates 2018 outcomes 3 professional knowledge

\section{Health promotion and illness prevention}

25. Newly qualified doctors must be able to apply the principles, methods and knowledge of population health and the improvement of health and sustainable healthcare to medical practice.

They must be able to:

1. Explain the concept of wellness or well-being as well as illness, and be able to help and empower people to achieve the best health possible, including promoting lifestyle changes such as smoking cessation, avoiding substance misuse and maintaining a healthy weight through physical activity and diet

2. Describe the health of a population using basic epidemiological techniques and measurements

3. Evaluate the environmental, social, behavioural and cultural factors which influence health and disease in different populations

4. Assess, by taking a history, the environmental, social, psychological, behavioural and cultural factors influencing a patient's presentation, and identify options to address these, including advocacy for those who are disempowered

5. Apply epidemiological data to manage healthcare for the individual and the community and evaluate the clinical and cost effectiveness of interventions

6. Outline the principles underlying the development of health, health service policy and clinical guidelines, including principles of health economics, equity and sustainable healthcare

7. Apply the principles of primary, secondary and tertiary prevention of disease, including immunisation and screening 


\section{Box 1. (Continued)}

8. Evaluate the role of ecological, environmental and occupational hazards in ill-health and discuss ways to mitigate their effects

9. Apply the basic principles of communicable disease control in hospital and community settings, including disease surveillance

10. Discuss the role and impact of nutrition to the health of individual patients and societies

11. Evaluate the determinants of health and disease and variations in healthcare delivery and medical practice from a global perspective and explain the impact that global changes may have on local health and well-being

Visibility of the PH specialty has always been variable within medical schools (Lyon, Hothersall, and Gillam, 2016). However, the morbidity and mortality due to COVID, the prevention and control measures put in place to reduce the spread of infection, as well as the direct impact on the delivery of medical education appears to have captured the 'sociological imagination' of medical students with more students volunteering to be involved in the prevention efforts as well as demonstrating a sharp rise of interest in understanding $\mathrm{PH}$ principles such as health inequalities and the social determinants of health.

In 2019, the UK Public Health Educators in Medical Schools (PHEMS) network updated its consensus statement mapping the core PH curriculum to the GMC Outcomes for Graduates, 2018 and the Faculty of Public Health (FPH) domains of PH practice (PHEMS, 2019). This statement highlights potential educational approaches and assessment methods for $\mathrm{PH}$, and opportunities for introducing $\mathrm{PH}$ throughout the clinical curriculum. The disproportionate impact of the COVID-19 pandemic on healthcare workers from Black, Asian and Minority Ethnic communities has further demonstrated the importance of integrating $\mathrm{PH}$ into the core undergraduate curriculum, rather than as an 'optional extra'. Furthermore, the Black Lives Matter movement in 2020 led to increased medical student awareness and discourse on human rights, racial discrimination, and health inequalities. The clinical relevance of the $\mathrm{PH}$ specialty has been brought into sharp focus as the national, regional and local responses to COVID have been led by $\mathrm{PH}$ specialists, epidemiologists and practitioners. This has required 
collaboration with clinical colleagues, healthcare providers and the community.

The PHEMS network, now a special interest group (SIG) of the FPH, organised a virtual event in March 2021 to bring together SIG members to discuss the impact of COVID and lockdown on $\mathrm{PH}$ education in medical schools. One of the most significant changes described was in the delivery of the teaching and the challenges of maintaining high-quality teaching during this rapid change. However, there were many examples of where these changes enhanced the delivery of core $\mathrm{PH}$ content, such as greater opportunities to access a range of partners including national/international health professions speakers and local community groups because of the pivot to online delivery of teaching. The COVID-19 response amplified the range of opportunities to deliver teaching across the breadth of the core $\mathrm{PH}$ curriculum and to integrate it with the clinical curriculum. Examples range from health protection (microbiological/clinical aspects of the virus/disease, developing clinical skills through supporting the mass vaccination programme and critical appraisal of the emerging trial evidence), health improvement (health inequalities both before and during the pandemic, community engagement approaches, impact of health policy on the determinants of health such as access to free school meals and furlough schemes) and health care PH (the local and national healthcare system response, evaluation of mass testing programmes and broader moral, ethical and legal implications of the response).

The question posed to SIG members on the day was whether these changes would result in a paradigm shift. GMC guidance (GMC 2018) already has an increased emphasis on the prevention and social medicine aspects of PH (Box 1). Will the pandemic and increased awareness of emerging infectious diseases, structural racism and health inequalities give PH more legitimacy now and genuinely translate into curriculum time, or will it just return to reinforcing the Cartesian mind and body of epidemiology and sociology? Medical school curricula that continue to focus on the biomedical model of health, and diagnosis and treatment of disease, rather than the complex interactions of the social determinants of health and disease are likely to produce medical graduates who are ill-equipped to treat patients in today's society (Rao et al., 2020).

Despite the difficulties, the pandemic could be thought of as providing a 'disruption' to the usual provision of medical education, creating an 
opportunity which schools can capitalise on to change or improve what was there before, to bring the substantial shifts in social priorities into medical education. This is an exciting time for medical education research to explore the innovations and their impact. For researchers and a new journal, the imperative is thus to address the right questions, and to create capacity to prioritise this at a time when research and evaluation may feel less of a priority than simply recovery. This opportunity may not come again, and it is our moral obligation to ensure that we learn all we can from it.

\section{REFERENCES}

General Medical Council (GMC), 2018. Outcomes for Graduates 2018. London: GMC. Available at: https://www.gmc-uk.org/-/media/ documents/dc11326-outcomes-for-graduates-2018_pdf-75040796. pdf [accessed 25 March 2021].

Gillam, S. and Bagade, A., 2006. Undergraduate public health education in UK medical schools - struggling to deliver. Medical Education, 40, pp. 430-436. https://doi.org/10.1111/j.1365-2929.2006.02444.x

Gillam, S., Rodrigues, V. and Myles, P., 2015. Public health education in UK medical schools - towards consensus. Journal of Public Health, 38(3), pp. 522-525. Available at: http://jpubhealth. oxfordjournals.org/content/early/2015/06/03/pubmed.fdv069.full. pdf+html [accessed 25 March 2021].

Lyon, A.K., Hothersall, E.J. and Gillam, S., 2016. Teaching public health in UK medical schools: 'things have improved: teaching no longer feels like an expensive hobby'. Journal of Public Health, 38(3), pp. e309-e315. Available at: https://academic.oup.com/ jpubhealth/article/38/3/e309/2239830 [accessed 25 March 2021].

PHEMS, 2019. Revised Undergraduate Public Health Curriculum for UK Medical Schools: A Consensus Statement. London: FPH. Available at: https://www.fph.org.uk/media/2685/phems-updatedconsensus-statement-2019-with-foreword_final.pdf [accessed 25 March 2021].

Rao, R., Hawkins, M., Ulrich, T., Gatlin, G., Mabry, G. and Mishra, C., 2020. The evolving role of public health in medical education. Frontiers in Public Health, 8, pp. 251. Available at: https://www.frontiersin .org/article/10.3389/fpubh.2020.00251 [accessed 30 March 2021]. 
PUBLIC HEALTH EDUCATION IN MEDICAL SCHOOLS - THE IMPACT OF THE COVID-19 PANDEMIC

\section{ACKNOWLEDGEMENT}

The authors would like to acknowledge both the Public Health Educators in Medical Education (PHEMS) group, and of the Behavioural and Social Sciences in Teaching in medicine (BeSST) group, both of whom contributed to the ideas expressed in the paper. 